\title{
Cyclin D1 G870A polymorphism: Association with uterine leiomyoma risk and in silico analysis
}

\author{
SAEEDEH SALIMI ${ }^{1,2}$, MAHNAZ SHAHRAKIPOUR ${ }^{3}$, AZAM HAJIZADEH $^{1,2}$, MOJGAN MOKHTARI $^{4,5}$, \\ MAHDIEH MOUSAVI $^{6}$, BATOOL TEIMOORI ${ }^{4,5}$ and MINOO YAGHMAEI $^{7}$

\begin{abstract}
${ }^{1}$ Cellular and Molecular Research Center, Zahedan University of Medical Sciences; ${ }^{2}$ Department of Clinical Biochemistry, School of Medicine, Zahedan University of Medical Sciences; ${ }^{3}$ Department of Biostatistics and Epidemiology, School of Public Health, Zahedan University of Medical Sciences; ${ }^{4}$ Department of Obstetrics and Gynecology, School of Medicine, Zahedan University of Medical Sciences; ${ }^{5}$ Pregnancy Health Research Center, Zahedan University of Medical Sciences, Zahedan, Sistan and Baluchestan 9816743175; ${ }^{6}$ Department of Biology, Faculty of Science, University of Zabol, Zabol, Sistan and Baluchestan 9861335856; ${ }^{7}$ Department of Obstetrics and Gynecology, School of Medicine, Shahid Beheshty University of Medical Sciences, Tehran 1985717413, Iran
\end{abstract}

Received October 21, 2016; Accepted November 24, 2016

DOI: $10.3892 /$ br. 2016.830

\begin{abstract}
Uterine leiomyoma (UL) is the most common benign tumor causing considerable morbidity during the reproductive years in women. Cyclin D1 (CCND1) is a cell cycle regulatory protein that is required for the G1 phase, and increased expression levels of this protein may affect tumorigenesis. The present study aimed to assess the possible effect of the CCND1 G870A polymorphism on UL susceptibility. A total of 154 women with UL and 197 healthy women who were age-, body mass index (BMI)- and ethnicity-matched were genotyped for the CCND1 G870A (rs9344) polymorphism using the polymerase chain reaction-restriction fragment length polymorphism method. The effects of G870A transition on the structure of mRNA and proteins of CCND1 was evaluated using bioinformatics tools. The frequency of the CCND1 870AA genotype was significantly higher in women with UL compared with the control subjects, and the risk of UL was 1.4-fold higher in women with the AA genotype when compared with the GG genotype before and after adjusting for age, BMI, and ethnicity [odds ratio (OR), 1.4; 95\% confidence interval $(\mathrm{CI}), 1.1-2(\mathrm{P}=0.02)]$. The frequency of $C C N D 1$ 870GA genotype was not significantly different between the two groups. The frequency of the CCND1 870A allele was significantly higher in the women with UL when compared with the control subjects ( 57 vs. $48 \%$; $\mathrm{P}=0.02$ ). The in silico
\end{abstract}

Correspondence to: Dr Minoo Yaghmaei, Department of Obstetrics and Gynecology, School of Medicine, Shahid Beheshty University of Medical Sciences, Shahid Arabi Street, Evin, Tehran 1985717413, Iran

E-mail: yaghmaeim@yahoo.com

Key words: cyclin D1, gene, polymorphism, uterine leiomyoma, in silico analysis revealed that the G870A transition may fundamentally alter the structure of the CCND1-mRNA. Thus, the CCND1 870AA genotype was associated with UL susceptibility in a sample of women from the southeast of Iran.

\section{Introduction}

Uterine leiomyomas (ULs) are benign tumors arising from smooth muscle cells of the uterine myometrium. ULs are characterized by the high smooth muscle cell (SMCs) proliferation and marked quantities of extracellular matrix proteins, predominantly collagen type I and III (1). UL development is considered a multistep and multi-factorial process, for which growth factors, cytokines, and ovarian steroid hormones are key in its formation and development (2). Different studies have shown that UL originate from somatic mutation in myometrial cells, which results in progressive loss of growth regulation (3). These tumors proliferate from a single progenitor cell and grow as abnormal clones of cells. ULs are monoclonal, and different leiomyomas arising within one uterus are not clonally linked (4). In addition, different rates of growth are due to chromosomal abnormalities within individual tumors (5). The occurrence of multiple ULs indicates a genetic predisposition for UL formation. There is a 2.5 -fold increased risk of developing UL in first-degree relatives of women with these types of tumor (6).

Abnormal cell proliferation is an important step in tumor development. There are various proteins in the cell cycle pathway that affect cell proliferation and tumorigenesis. Among these proteins, cyclins are particularly important in this process (7). Cyclins are a family of proteins that affect cell progression through the cell cycle by triggering cyclin-dependent kinase (CDK) enzymes. Cyclin D1 (CCND1) is a key regulatory protein that binds to CDK4 and CDK6 enzymes, and functions during the transition from the G1 to the $\mathrm{S}$ phase (8). Increased expression of the CCND1 gene may disrupt normal cell cycle control, and prompt cells to 
transition from the $\mathrm{G}_{1} / \mathrm{S}$ checkpoint of the cell cycle resulting in tumor formation (8). Various studies have demonstrated that overexpression of the CCND1 protein is associated with cell proliferation and different types of cancer, including endometrial (9), cervical (10), lung (11) and breast (12) cancer.

Betticher et al (13) described a $\mathrm{G}$ to A single nucleotide polymorphism (G870A) at the exon4/intron4 splicing region of the $C C N D 1$ gene. The A allele has a longer half-life than the $\mathrm{G}$ allele, which is hypothesized to increase CCND1 levels resulting in the proliferation of abnormal cells and the escape of these cells from apoptosis (13). To the best of our knowledge, there is only one published report that has indicated the effect of the $C C N D 1$ G870A polymorphism on UL susceptibility in obese Korean women (14), although there are different reports regarding the association between CCND1 gene polymorphisms, and tumors and cancers.

Thus, the present study aimed to assess the possible association between the $C C N D 1$ G870A polymorphism and UL in a southeastern Iranian population.

\section{Materials and methods}

Study population. A total of 154 Iranian women (aged $37.9 \pm 8.9$ years) with UL in their pre-menopause stage who had undergone myomectomy or hysterectomy and 197 Iranian healthy women (aged $37.5 \pm 5.5$ years) in their pre-menopause stage were enrolled in the study. All individuals were recruited from the Department of Obstetrics and Gynecology of Ali-ebn-Abitaleb Educational Hospital of Zahedan University of Medical Sciences (Zahedan, Iran) from July 2012 to October 2013. UL was confirmed pathologically in all of the women. The control group was selected from women that were referred for routine yearly check-ups and undergone the Pap smear test. The UL and control groups were matched with respect to age, body mass index (BMI), and ethnicity (Fars or Balouch). Upon sonography or examination, the participants in the control group exhibited no evidence of UL and their Pap smear test was negative. Women with systemic disease and history of malignancy were excluded from the present study. The participants provided their informed consent prior to participating in the study and the study protocol was approved by the Ethics Committee of Zahedan University of Medical Sciences (Zahedan, Iran) and conducted in accordance with the Declaration of Helsinki.

Genomic DNA extraction and genotyping. Venous blood ( $2 \mathrm{ml})$ was drawn from each participant. Genomic DNA was isolated from $200 \mu$ l EDTA-treated whole blood using the salting out phenol chloroform method and stored at $20^{\circ} \mathrm{C}$ until analysis. Polymerase chain reaction-restriction fragment length polymorphism (PCR-RFLP) was performed for genotyping the CCND1 G870A polymorphism.

The 167-bp fragment containing the CCNDI G870A polymorphism (rs9344) was amplified using primers as previously described (13). The PCR conditions were as follows: 6 min at $95^{\circ} \mathrm{C}$ followed by 30 cycles of denaturation at $96^{\circ} \mathrm{C}$ for $30 \mathrm{sec}$, annealing at $58^{\circ} \mathrm{C}$ for $30 \mathrm{sec}$, extension at $72^{\circ} \mathrm{C}$ for $30 \mathrm{sec}$, and a final extension at $72^{\circ} \mathrm{C}$ for $6 \mathrm{~min} . \mathrm{Nci}$ I restriction enzyme (Fermentas; Thermo Fisher Scientific, Inc., Pittsburgh, PA, USA) was used to digest the PCR products at $37^{\circ} \mathrm{C}$ overnight.
Table I. Clinical and demographic characteristics of the UL women and control group subjects.

\begin{tabular}{|c|c|c|c|}
\hline Parameter & $\begin{array}{l}\text { UL women } \\
(\mathrm{n}=154)\end{array}$ & $\begin{array}{l}\text { Control } \\
\text { subjects } \\
(\mathrm{n}=197)\end{array}$ & P-value \\
\hline Age (years) & $37.9 \pm 8.9$ & $37.5 \pm 5.5$ & 0.625 \\
\hline Body mass index $\left(\mathrm{kg} / \mathrm{m}^{2}\right)$ & $25.6 \pm 5.3$ & $25.3 \pm 4.6$ & 0.588 \\
\hline Parity (n) & $3.4 \pm 2.1$ & $3.5 \pm 2.6$ & 0.708 \\
\hline Age of menarche (years) & $13.4 \pm 1.3$ & $13.2 \pm 1.6$ & 0.123 \\
\hline Duration of menses (days) & $6.0 \pm 1.6$ & $5.9 \pm 1.6$ & 0.817 \\
\hline Menstrual cycle (days) & $28.6 \pm 2.2$ & $28.5 \pm 2.9$ & 0.686 \\
\hline Bleeding, n (\%) & $93(60.0)$ & $9(4.5)$ & $<0.001$ \\
\hline Pain, n (\%) & $43(28.0)$ & $13(7.0)$ & $<0.001$ \\
\hline
\end{tabular}

Unless otherwise stated, values are presented as means \pm standard deviation. UL, uterine leiomyoma.

The digested products were electrophoresed on 3\% agarose gel at $90 \mathrm{~V}$ for $80 \mathrm{~min}$ and visualized by ethidium bromide staining.

In silico analysis. DNA sequences of CCNDI gene were obtained from The National Center for Biotechnology Information (accession no. CR536538.1). The coding sequence domain of CCND1 was translated using the ExPASy server (http://web.expasy.org/translate/). Mode 2 of the RNAsnp (http://rth.dk/resources/rnasnp/) was used to evaluate the effects of G870A transition on the CCND1-mRNA structure. RNAsnp is a server that predicts the effect of single nucleotide polymorphisms (SNPs) on the local RNA secondary structure based on the RNA folding algorithms (15). In the graphic summary of the RNAsnp report, the local region, which detects maximum structural change, is colored according to the $\mathrm{P}$-value. If $\mathrm{P}>0.2$ the region is colored black, indicating that the structural change that occurred is not significant.

Statistical analysis. All statistical analyses were conducted using SPSS version 20 (IBM SPSS, Armonk, NY, USA). Demographic characteristics of the groups were analyzed through independent Student's t-test or Fisher's exact test. Logistic regression analysis assessed the independent effect of each polymorphism risk on UL. Odds ratios (ORs) and 95\% confidence intervals (CIs) were considered for significant allelic and genotypic associations. Using the $\chi^{2}$ test, genotype frequencies of the polymorphism were evaluated for deviation from Hardy-Weinberg equilibrium. $\mathrm{P}<0.05$ was considered to indicate statistically significant differences.

\section{Results}

Study characteristics. The demographic characteristics of the women with UL and the control group subjects are provided in Table I. The age, BMI and parity (total number of births after 20 weeks) were not significantly different between the UL women and control subjects. No significant differences in menstrual histories, including age of menarche, menstrual 
Table II. Comparison of the genotypic and allelic frequency of the cyclin D1 (rs9344) polymorphism in UL women and the control group.

\begin{tabular}{lcccccc}
\hline Polymorphism & $\begin{array}{c}\text { Control subjects } \\
(\mathrm{n}=197)\end{array}$ & $\begin{array}{c}\text { UL women } \\
(\mathrm{n}=154)\end{array}$ & P-value & OR (95\% CI) & P-value $^{\mathrm{a}}$ & ${\text { OR }(95 \% \mathrm{CI})^{\mathrm{a}}}$ \\
\hline Cyclin D1 (rs9344) & & & & & & \\
GG, n (\%) & $50(25.4)$ & $31(20)$ & - & 1 & - & 1 \\
GA, n (\%) & $105(53.3)$ & $70(45.5)$ & 0.79 & $0.9(0.5-1.6)$ & 0.89 & $1(0.6-1.7)$ \\
AA, n (\%) & $42(21.3)$ & $53(34.5)$ & 0.02 & $1.4(1.1-1.9)$ & 0.02 & $1.4(1.1-2)$ \\
Allele & & & & & - & - \\
G, n (\%) & $205(52)$ & $132(43)$ & - & 1 & - \\
A, n (\%) & $189(48)$ & $176(57)$ & 0.02 & $1.5(1.1-2)$ & - & - \\
\hline
\end{tabular}

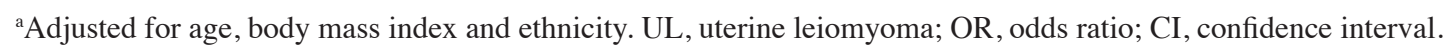
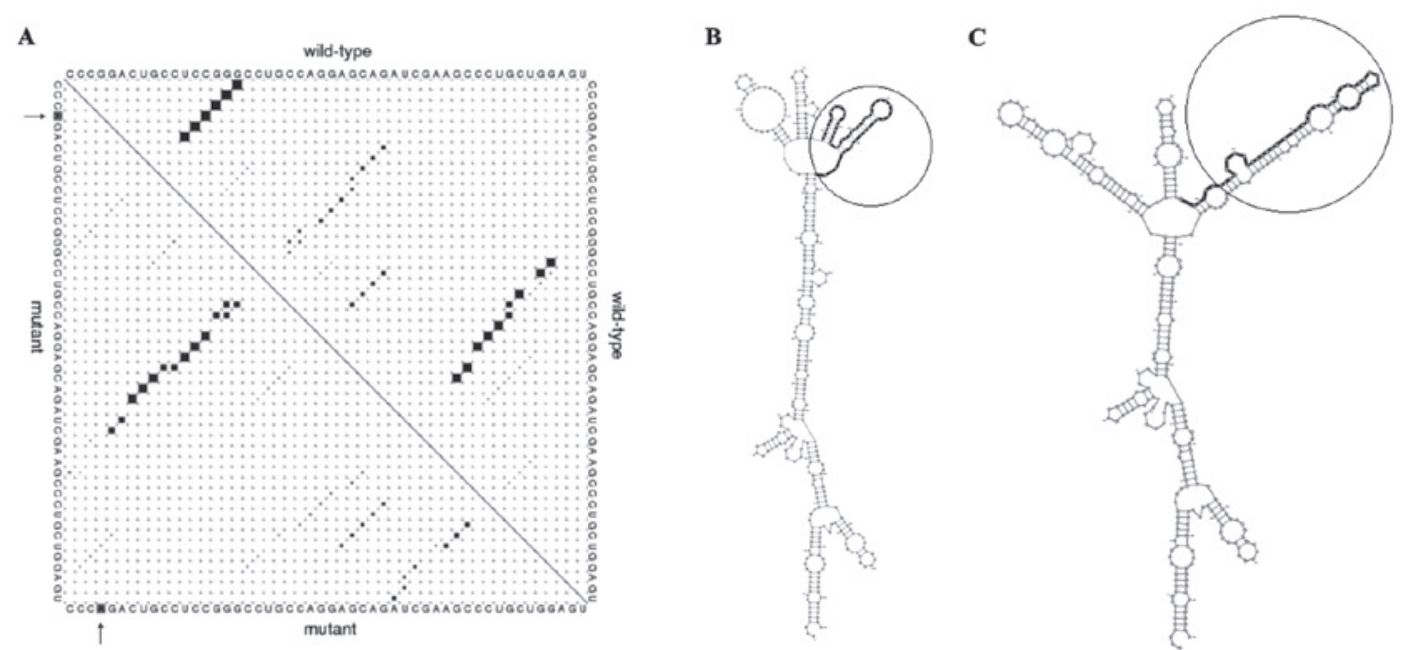

Figure 1. G870A transition effects on CCND1-mRNA were analyzed by RNAsnp. (A) Local region with maximum differences in wild-type and mutant CCND1-mRNA. Base pair probabilities of the local region (523-888) detected with the maximum differences depicted. The upper and lower triangle of the matrix represents the base pair probabilities of wild-type and mutant sequences, respectively. The mutated nucleotide is presented by the arrow. (B) The optimal secondary structure of global wild-type sequence (523-888; circled) with a minimum free energy of $-138.70 \mathrm{kcal} / \mathrm{mol}$. (C) The optimal secondary structure of global mutant sequence (523-888; circled) with a minimum free energy of $-134.50 \mathrm{kcal} / \mathrm{mol}$.

cycle and duration of menses were identified among the women with UL and the control subjects. The frequency of bleeding and pain was observed to be more frequent in women with UL $(\mathrm{P}<0.0001)$.

Genotypes and UL risk. The allelic and genotypic frequencies of the CCND1 G870A polymorphism in women with UL and the control subjects are presented in Table II. The CCNDI G870A polymorphism conformed with Hardy-Weinberg equilibrium $(\mathrm{P}>0.05)$. The frequency of $\mathrm{GG}, \mathrm{GA}$, and $\mathrm{AA}$ genotypes were $20,45.5$ and $34.5 \%$, respectively in the women with UL, and 25.4, 53.3 and $21.3 \%$, respectively in the control group. The frequency of the AA genotype was significantly higher in women with UL compared with the control subjects, although the frequency of the GA genotype was not statistically different between the two groups before and after adjusting for age, BMI, and ethnicity (dominant model); thus, the risk of UL was 1.4-fold greater in women with the AA genotype when compared to the GG genotype before and after adjusting for age, BMI, and ethnicity (OR, 1.4; 95\% CI, 1.1-2; $\mathrm{P}=0.02)$. Furthermore, the frequency of the CCND1 870A allele was significantly higher in the women with UL when compared with the control subjects (57 vs. $48 \% ; \mathrm{P}=0.02$ ).

Structural analysis. In silico analysis indicated that rs 9344 $(\mathrm{G}>\mathrm{A})$ polymorphism is located at positions 950,723 and 241 of the transcript, CDS and CCND1 protein, respectively, which alters the CCG codon to CCA. Since these two codons translated to proline, this mutation does not change the protein features of CCND1, including molecular weight, isoelectric point and physicochemical properties (data not shown). Predicting 870AA effects on the local RNA secondary structure of CCND1 revealed that the SNPs cause fundamental changes to the secondary structure of mRNA (distance, $0.1922 ; \mathrm{P}=0.1488 ; \mathrm{P}<0.2$ indicates significant structural change) (Fig. 1). The base pair probabilities of the local region detected with maximum differences locate between base pairs 523 and 888 (Fig. 1A). Furthermore, the optimal 
secondary structure of global wild-type and mutant sequences are presented in Fig. 1B and C.

\section{Discussion}

In the current study, a higher frequency of the CCND1 870AA genotype was identified in women with UL when compared to the GG genotype, thus, CCND1 870AA homozygous polymorphism may increase the risk of UL.

CCND1 is an important protein in regulation of the cell cycle, causing the transition from the $\mathrm{G}_{1}$ to the $\mathrm{S}$ phase of the cell cycle. CCND1 regulates cell cycle progression by activating CDK4 and CDK6 $(7,8)$ and the activated enzymes phosphorylate the retinoblastoma $(\mathrm{Rb})$ protein. The phosphorylation of $\mathrm{Rb}$ releases the transcriptional factor, E2F, which subsequently triggers numerous downstream genes that are necessary for the cell cycle (16).

Altered expression levels of cell cycle regulatory proteins, such as CCND1, are associated with cell proliferation and are present in different types of human tumor and cancer, including endometrial $(9,17)$ and cervical $(18)$ cancer, and endometriosis and adenomyosis (19). An elevated expression level of the CCND1 protein was observed in leiomyomas compared with the adjacent myometrium during all menstrual cycle phases in a study by Kovács et al (20). There are several genetic variants in cell cycle regulatory proteins, which affect protein expression and prevent or stimulate cell proliferation, increasing the susceptibility to tumor development. Hence, molecular epidemiologic studies are considered to be useful strategies for investigating the association between genetic variations and tumorigenesis (21).

In 1995, Betticher et al (13) described a G870 to A SNP at codon 242 on exon 4 in the CCND1 gene, which affected splicing of the CCND1 transcript. CCND1 mRNA exhibits different splicing, in which translation of the transcripts produces various proteins with dissimilar c-terminal domains. The CCND1 G870A polymorphism may control splicing of the transcripts, and the $870 \mathrm{~A}$ allele generates the truncated transcript, encoding a protein with a longer half-life; however, the G870 allele produces the full transcript (13). Therefore, different studies have considered the CCNDI 870A allele as a susceptible variant in tumorigenesis. Although, there are numerous reports on the correlation between the CCNDI G870A polymorphism and different types of cancer $(22,23)$, to the best of our knowledge, there is only one published study regarding the association between the CCND1 G870A polymorphism and UL susceptibility in obese Korean women (14). In 2008, Han et al (14) demonstrated that the AA genotype and A allele of the $C C N D 1 \mathrm{G} 870 \mathrm{~A}$ polymorphism may increase UL risk in Korean women with a BMI $>25 \mathrm{~kg} / \mathrm{m}$ (2), confirming the results of the current study.

Although G870A transition does not change amino acid substitution in the CCND1 protein, this SNP may alter the CCND1-mRNA structure and function. The mRNAs containing different nucleotides at an SNP position may differ in their interactions with cellular molecules involved in mRNA transport, maturation, translation or degradation (24). Analysis of the effects of G870A transition on the secondary structure of mRNA indicated that this SNP may alter the structure and minimum free energy of mRNA. Therefore, G870A may alter
CCND1 gene expression levels. Although the current results of in silico analysis improve our understanding of the effects of G870A on the CCND1 mRNA, further studies are required to evaluate the influence of this SNP on UL susceptibility.

There were certain limitations of the current study. As the conclusion is based on a small number of patients and control subjects, the statistical power is not considered to be particularly high. In addition, the study would be more valuable if myomatous and intact adjacent tissue samples had been investigated. Finally, as there are different ethnic groups in Iran, further studies are required with greater sample sizes from each ethnic group to confirm or refute our findings.

In conclusion, the findings of the present study revealed that the CCND1 870AA genotype and CCND1 870A allele were associated with a predisposition for UL, and that this polymorphism may be a risk factor for UL. Further studies with greater sample sizes from different populations are required to validate the current findings.

\section{Acknowledgements}

The present study was extracted from an MS thesis (registered no. 6169) at Zahedan University of Medical Sciences (Zahedan, Iran). The authors would like to thank the Deputy of Research Affairs at Zahedan University of Medical Sciences for funding this project.

\section{References}

1. Stewart EA, Friedman AJ, Peck K and Nowak RA: Relative overexpression of collagen type I and collagen type III messenger ribonucleic acids by uterine leiomyomas during the proliferative phase of the menstrual cycle. J Clin Endocrinol Metab 79: 900-906, 1994.

2. Barbarisi A, Petillo O, Di Lieto A, Melone MA, Margarucci S, Cannas M and Peluso G: 17-beta estradiol elicits an autocrine leiomyoma cell proliferation: Evidence for a stimulation of protein kinase-dependent pathway. J Cell Physiol 186: 414-424, 2001.

3. Jeon YT, Kim JW, Park NH, Song YS, Kang SB and Lee HP: DNA repair gene XRCC1 Arg399Gln polymorphism is associated with increased risk of uterine leiomyoma. Hum Reprod 20: 1586-1589, 2005.

4. Gittenberger-de Groot AC, DeRuiter MC, Bergwerff M and Poelmann RE: Smooth muscle cell origin and its relation to heterogeneity in development and disease. Arterioscler Thromb Vasc Biol 19: 1589-1594, 1999.

5. Medikare V, Kandukuri LR, Ananthapur V, Deenadayal M and Nallari P: The genetic bases of uterine fibroids; a review. J Reprod Infertil 12: 181-191, 2011

6. Vikhlyaeva EM, Khodzhaeva ZS and Fantschenko ND: Familial predisposition to uterine leiomyomas. Int J Gynaecol Obstet 51: 127-131, 1995.

7. Galderisi U, Jori FP and Giordano A: Cell cycle regulation and neural differentiation. Oncogene 22: 5208-5219, 2003.

8. Sherr CJ: Cancer cell cycles. Science 274: 1672-1677, 1996.

9. Shevra CR, Ghosh A and Kumar M: Cyclin D1 and Ki-67 expression in normal, hyperplastic and neoplastic endometrium. J Postgrad Med 61: 15-20, 2015.

10. Bae DS, Cho SB, Kim YJ, Whang JD, Song SY, Park CS, Kim DS and Lee JH: Aberrant expression of cyclin D1 is associated with poor prognosis in early stage cervical cancer of the uterus. Gynecol Oncol 81: 341-347, 2001.

11. K Ayed A and Adesina A: Prognostic significance of cyclin D1 expression in resected stage I, II non-small cell lung cancer in Arabs. Interact Cardiovasc Thorac Surg 5: 47-51, 2006.

12. Abramson VG, Troxel AB, Feldman M, Mies C, Wang Y, Sherman L, McNally S, Diehl A and Demichele A: Cyclin Dlb in human breast carcinoma and coexpression with cyclin D1a is associated with poor outcome. Anticancer Res 30: 1279-1285, 2010. 
13. Betticher DC, Thatcher N, Altermatt HJ, Hoban P, Ryder WD and Heighway J: Alternate splicing produces a novel cyclin D1 transcript. Oncogene 11: 1005-1011, 1995.

14. Han SS, No JH, Jeon YT, Kim JW, Park NH, Song YS, Kang SB and Lee HP: Association of cyclin D1 G870A polymorphism with uterine leiomyoma in women whose body mass index values are above $25 \mathrm{~kg} / \mathrm{m} 2$. Hum Reprod 23: 525-529, 2008.

15. Sabarinathan R, Tafer H, Seemann SE, Hofacker IL, Stadler PF and Gorodkin J: The RNAsnp web server: Predicting SNP effects on local RNA secondary structure. Nucleic Acids Res 41: W475-W479, 2013

16. Mallya SM and Arnold A: Cyclin D1 in parathyroid disease. Front Biosci 5: D367-D371, 2000.

17. Catalano S, Giordano C, Rizza P, Gu G, Barone I, Bonofiglio D, Giordano F, Malivindi R, Gaccione D, Lanzino M, et al: Evidence that leptin through STAT and CREB signaling enhances cyclin D1 expression and promotes human endometrial cancer proliferation. J Cell Physiol 218: 490-500, 2009.

18. Li HY, Xu Q, Zhu T, Zhou JH, Deng DR, Wang SX, Lu YP and Ma D: Expression and clinical significance of Pin1 and Cyclin D1 in cervical cancer cell lines and cervical epithelial tissues. Ai Zheng 25: 367-372, 2006 (In Chinese).

19. Goumenou AG, Matalliotakis IM, Tzardi M, Fragouli IG, Mahutte NG and Arici A: p16, retinoblastoma (pRb), and cyclin D1 protein expression in human endometriotic and adenomyotic lesions. Fertil Steril 85 (Suppl 1): 1204-1207, 2006.
20. Kovács KA, Oszter A, Göcze PM, Környei JL and Szabó I: Comparative analysis of cyclin D1 and oestrogen receptor (alpha and beta) levels in human leiomyoma and adjacent myometrium. Mol Hum Reprod 7: 1085-1091, 2001.

21. Abreu Velez AM and Howard MS: Tumor-suppressor Genes, Cell Cycle Regulatory Checkpoints, and the Skin. N Am J Med Sci 7: 176-188, 2015.

22. Dai X, Zhang X, Wang B, Wang C, Jiang J and Wu C: Association Between Polymorphism rs678653 in Human Cyclin D1 Gene (CCND1) and Susceptibility to Cancer: A Meta-Analysis. Med Sci Monit 22: 863-874, 2016.

23. Yang $\mathrm{M}$, Zhu $\mathrm{H}, \mathrm{Hu} \mathrm{T}$, Liu $\mathrm{S}$ and Wang $\mathrm{H}$ : Association of CCND1 gene polymorphism with cervical cancer susceptibility in Caucasian population: A meta-analysis. Int J Clin Exp Med 8: 12983-12988, 2015.

24. Shen LX, Basilion JP and Stanton VP Jr: Single-nucleotide polymorphisms can cause different structural folds of mRNA. Proc Natl Acad Sci USA 96: 7871-7876, 1999. 